\title{
Electronically Tunable Antenna Pair and Novel RF Front-End Architecture for Software-Defined Radios
}

\author{
Sung-Hoon Oh \\ Department of Electrical Engineering, Arizona State University, Tempe, AZ 85287, USA
}

Email: sung-hoon.oh@asu.edu

James T. Aberle

Department of Electrical Engineering, Arizona State University, Tempe, AZ 85287, USA Email: aberle@asu.edu

\author{
Sreeraman Anantharaman \\ Department of Electrical Engineering, Arizona State University, Tempe, AZ 85287, USA \\ Email:sreeram@asu.edu@asu.edu
}

\section{Kentaro Arai}

Department of Electrical Engineering, Arizona State University, Tempe, AZ 85287, USA Email: bluewaterken@hotmail.com

\author{
Han Liang Chong \\ Department of Electrical Engineering, Arizona State University, Tempe, AZ 85287, USA \\ Email: hanchong@asu.edu
}

\section{Seng Chee Koay}

Department of Electrical Engineering, Arizona State University, Tempe, AZ 85287, USA Email:sun_devil2000@hotmail.com

Received 1 February 2004; Revised 14 September 2004

\begin{abstract}
This paper proposes a novel RF front-end architecture for software-defined radios (SDRs) based on an electronically tunable antenna pair controlled by an antenna control unit (ACU) consisting of field effect transistor (FET) switches and a field programmable gate array (FPGA). The fundamental gain-bandwidth limitations of electrically small antennas prevent a small antenna from having high efficiency and wide bandwidth simultaneously. In the age of miniaturization, especially in the wireless communication industries, a promising solution to this limitation is to introduce reconfigurable antennas that can be tuned electronically to different frequency bands with both high efficiency and narrow instantaneous bandwidth. This reconfigurable antenna technology not only simplifies current RF front-end architectures, but can be reprogrammed on demand to transmit and receive RF signals in any desired frequency band. This novel RF front-end architecture implemented by a reconfigurable antenna pair can help realize SDRs.
\end{abstract}

Keywords and phrases: antenna, ACU, FET, FPGA, RF front end, SDRs.

\section{INTRODUCTION}

Software-defined radios (SDRs) are considered to be the ultimate solution for the future of wireless communications.

This is an open access article distributed under the Creative Commons Attribution License, which permits unrestricted use, distribution, and reproduction in any medium, provided the original work is properly cited.
However the application of SDRs over wide frequency bands is constrained at the RF front end due to analog hardware parts, especially the antenna [1]. The gain-bandwidth limitation of electrically small antennas is a fundamental law of physics that limits the ability of the wireless system engineer to simultaneously reduce the size of an antenna while increasing its bandwidth and efficiency [2]. A revolutionary approach to circumventing this limitation is to construct 


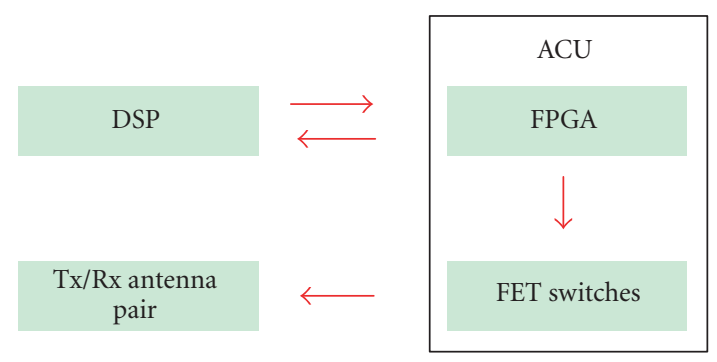

FIGURE 1: Block diagram of the reconfigurable antenna pair controlled by DSP.

a small, highly efficient antenna with a narrow instantaneous bandwidth that can be electronically tuned over wide frequency bands.

The reconfigurability of the antennas is achieved by altering the geometry of the antenna electronically based on the frequency information received by an antenna control unit (ACU). The ACU consists of the field programmable gate array (FPGA) and field effect transistor (FET) switches. The FPGA will act as a microcontroller to toggle FET switches to effectively change the electrical length of the antennas. The block diagram of a reconfigurable antenna pair used in an SDR is shown conceptually in Figure 1. Introducing an electronically tunable antenna pair that receives commands from the radio's digital signal processor (DSP) is a promising solution for realizing multiband radios and SDRs.

Furthermore, the narrow instantaneous frequency response of the reconfigurable antenna pair would greatly reduce the need for additional analog filtering in the RF front end, which will also reduce the size, cost, and power requirements of wireless mobile devices. Such an antenna pair would reject undesired interferers with a minimum degradation of the desired signal's signal-to-noise ratio (SNR). In this paper, it is found that selectivity of the reconfigurable antenna pair provides filter-like performance so that duplexing, diplexing, and bandpass image rejection functions are absorbed into the antenna. In addition, a novel RF front-end architecture which is simpler and more cost-effective than current RF architectures is proposed to improve the performance of the RF front end and demonstrate the feasibility of SDR applications.

\section{RECONFIGURABLE ANTENNA PAIR}

In this section, we discuss the design of an electronically tunable shorted patch antenna (SPA) controlled by an ACU consisting of FET switches and FPGA.

\subsection{Shorted patch antenna (SPA)}

SPA is one of the most promising antennas for wireless mobile devices such as mobile handsets because of their compactness, conformability, low-cost, and omni-directional patterns [3]. The SPA is loaded with small and low-cost variable capacitors at the radiating edge to effectively change the electrical length of the antenna. The required physical size of SPA for resonance is also reduced by the capacitor loading.

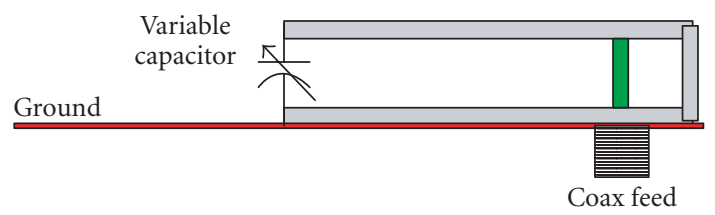

FIGURE 2: Geometry of SPA for reconfigurable antenna implementation.

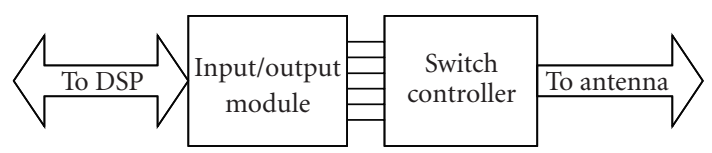

FIGURE 3: Block diagram outlining the functionality of the FPGA for ACU.

The geometry of the SPA for the electronically tunable antenna is shown in Figure 2. The SPA is designed with a layer of copper placed above a ground plane separated by a substrate layer. The shorting edge is accomplished by connecting one side of the square radiating patch to the ground plane. To feed the SPA to the ground plane, the outer conductor of the SMA connector is connected to the ground plane while the inner conductor is connected to the patch.

It is necessary to model the antenna as a two-port device to account for the effect of its frequency response on the system. It is shown in [4] that the forward transmission coefficient (i.e., the scattering parameter, $\mathrm{S}_{21}$ ) of the antenna is equal to its total efficiency, which includes radiation efficiency and mismatch loss. Both copper loss and tuning circuit loss contribute to reduced radiation efficiency and also affect the bandwidth. This two-port equivalent circuit of the SPA is invaluable for calculating the practical limitations on bandwidth, size, efficiency, and the effects that losses in the tuning circuits have on these quantities.

\subsection{Antenna control unit (ACU)}

The ACU introduced in this paper employs both analog and digital technology. FET switches are adopted in order to change the electrical dimension within each antenna and to tune the antenna pair between GSM850(US Cellular)/GSM900 and PCS/DCS bands. The on/off status of single-pole double-throw (SPDT) FET switches are controlled by the FPGA based on frequency information received from the DSP. Employing the FPGA for the ACU provides the end users with complete control over the tunability of antenna.

An overall perspective view of the system level design is discussed in this section. The ACU is divided into two modules as outlined in the schematic shown in Figure 3. The input/output module receives commands from the radio's DSP and then translates them into a necessary format for the use of the switch controller. The hardware description language (HDL) code residing on the input/output module of the FPGA will be responsible for the initial hand shaking to accept data or serve as the end of the line for the transmitted data. For demonstration purposes, this module is programmed to receive frequency information in the RS-232 


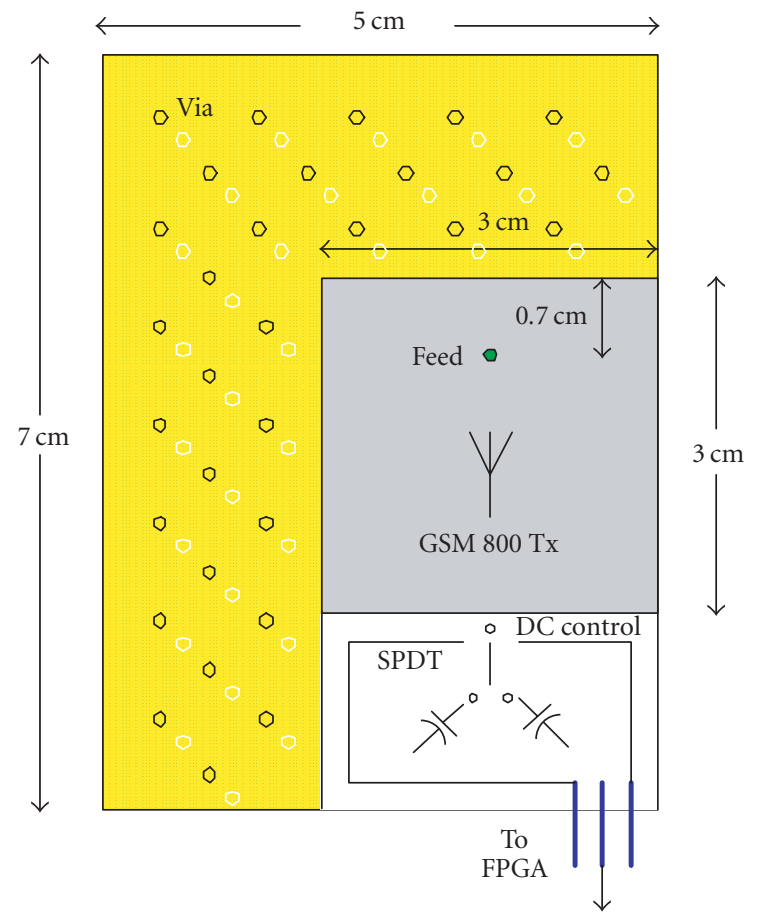

FIGURE 4: Dimensions of the tunable GSM 850/900 SPA mounted on a PCB with ACU.

asynchronous serial format and convert the serial data into parallel data format.

The switch controller module receives parallel frequency data from the input/output module and generates required switch control signals to reconfigure the antenna pair. For SDR applications, this module can be updated anytime the RF front-end device needs to change to an application involving different frequency bands.

\subsection{Tunable SPA design for GSM $850 / 900$}

An electronically tunable SPA covering the transmit portions of the US cellular (824-849 MHz) and GSM 900 (890$915 \mathrm{MHz}$ ) bands was designed and fabricated in order to demonstrate the feasibility of the reconfigurable antenna pair for mobile handsets. The dimensions of the antenna and the printed circuit board upon which it is mounted are shown in Figure 4. A picture of the physical hardware that was constructed is shown in Figure 5. The antenna has length, width, and height of $30 \times 30 \times 7 \mathrm{~mm}$, respectively. The tuning circuit consists of variable capacitors and an SPDT FET switch that is controlled by the ACU. The SPA is electronically tuned between the GSM 850 and GSM 900 bands based on the frequency information provided by the user to the FPGA. The measured return loss for both states is presented in Figure 6 . The return loss of the antenna is a measure of impedance mismatch between the antenna input and its $50 \Omega$ feeding line. The bandwidth of the antenna is, in general, determined by determining the frequency range over which the return loss is less than $-10 \mathrm{~dB}$. As shown in Figure 6, the bandwidth of the return loss response in each state is about equal to the $25 \mathrm{MHz}$ bandwidth of the transmit portions of the US cellular and GSM 900 bands.

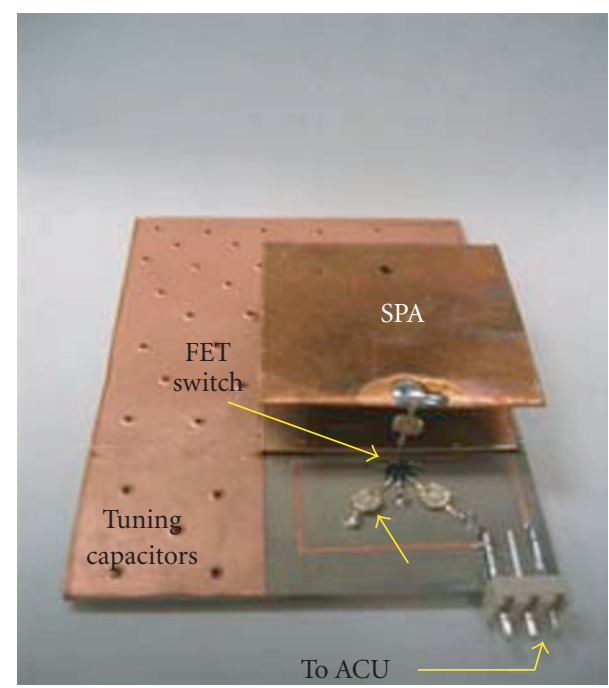

Figure 5: Photo of the fabricated tunable GSM 850/900 SPA.

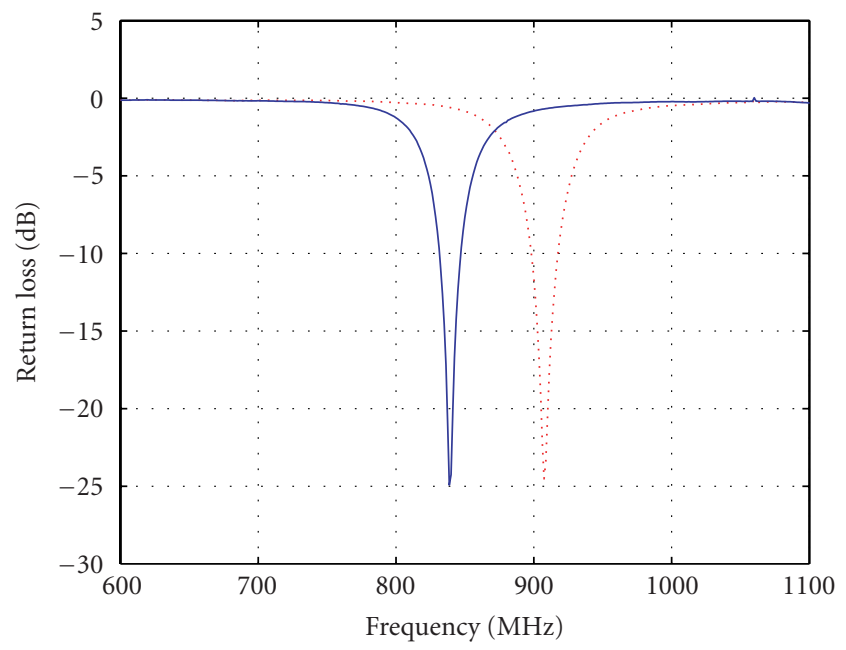

GSM 900 Tx

- GSM $850 \mathrm{Tx}$

FIGURE 6: Measured return loss of the reconfigurable SPA between GSM 850 and GSM 900 bands.

\subsection{Tunable SPA pair for GSM $850 / 900, D C S$, and PCS}

After successfully fabricating a single SPA, two antenna pairs consisting of four individual SPAs are designed to cover all of mobile communication bands: GSM850/900, DCS, and PCS. The layout of the prototype is shown in Figure 7. The tunability between GSM and PCS/DCS bands is achieved by using two different sizes of SPAs, the larger for GSM and the smaller for PCS/DSC bands, and by switching the RF signal paths. The antenna pair for GSM bands has length, width, and height of $30 \times 30 \times 7 \mathrm{~mm}$ and $20 \times 20 \times 6 \mathrm{~mm}$ for PCS/DCS bands. In order to minimize the coupling and interference between each antenna pair, the antennas are arranged orthogonally relative to each other. Coupling of less than 

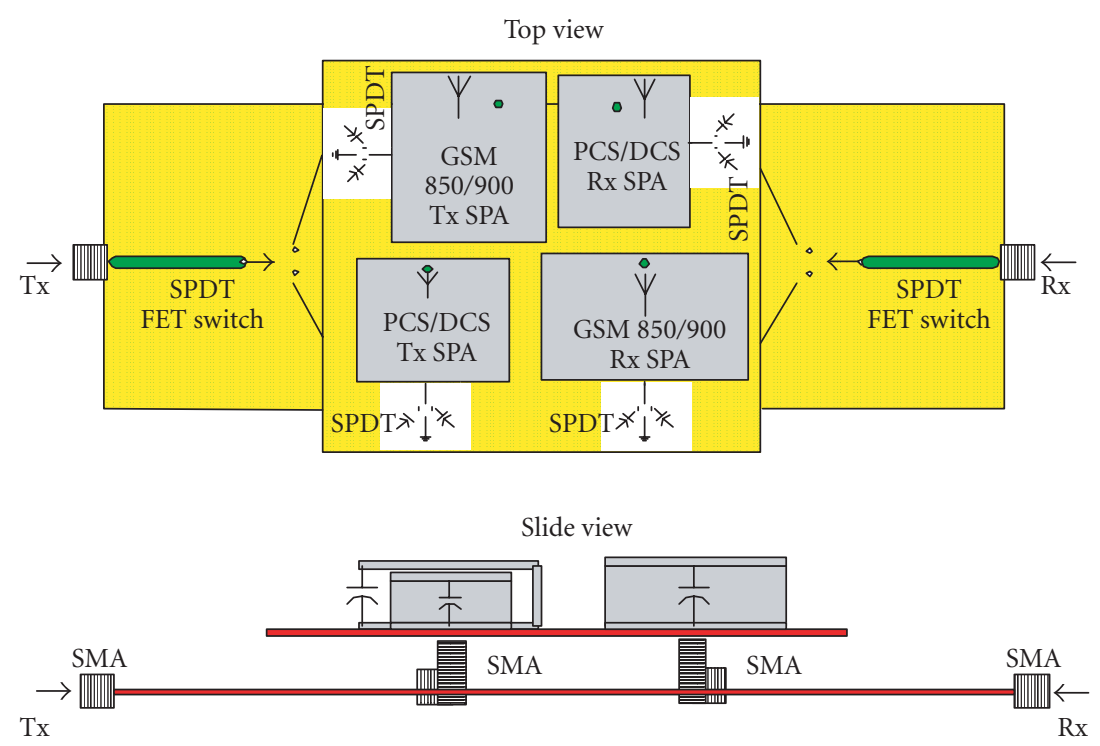

FIGURE 7: Prototype of tunable antennas for GSM850/900, DCS, and PCS.

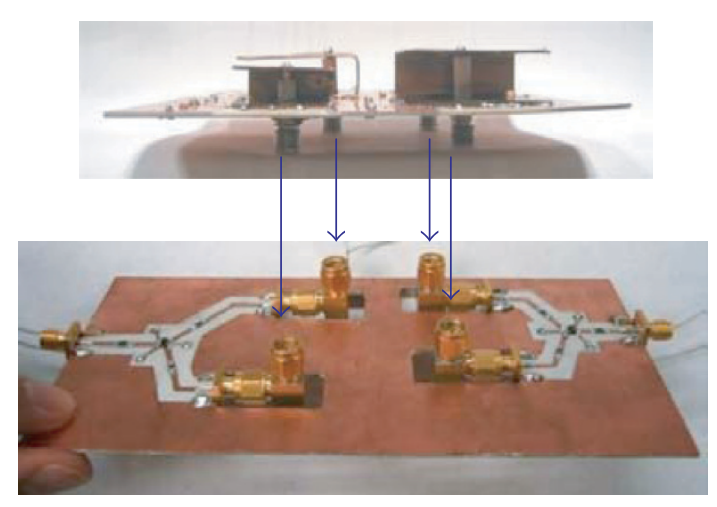

FIgURE 8: Photo of the prototype of tunable antennas for GSM850/900, DCS, and PCS.

$20 \mathrm{~dB}$ between the Tx and $\mathrm{Rx}$ antennas was achieved. The tuning techniques for each SPA are described in Section 2.3. The picture of the prototype is shown in Figure 8.

\section{ELECTRONICALLY TUNABLE ANTENNA IMPLEMATATION}

With the SPA pairs fully fabricated and tested as well as the ACU programmed and implemented, the setup shown in Figure 9 was assembled to evaluate the performance of the electronically tunable antenna pairs. For demonstration purposes, the DSP was emulated by a graphical user interface which accepts frequency information from a user and converts the frequency information into the required series binary format for RS-232 communication. The SPA pair is electronically tuned between GSM 850 and GSM 900, and the measured return loss for each state is presented in Figure 10.

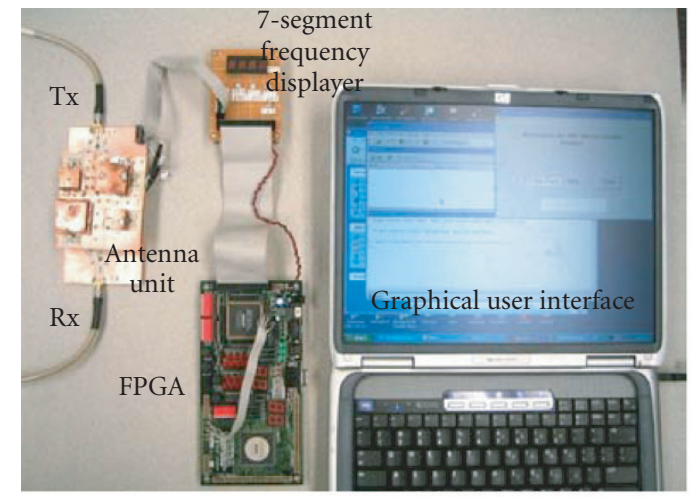

Figure 9: Test setup for demonstrating the electronically tunable antennas.

\section{NOVEL RF FRONT END FOR SDRs}

Based on the careful review of antenna characteristics and experimental results revealing that the return loss of an SPA resembles the frequency response of a bandpass filter, we became interested in determining if the frequency responses of the $\mathrm{Tx} / \mathrm{Rx}$ antenna pair could be used to eliminate or reduce the requirements on the RF filters required in a wireless transceiver. Separate $\mathrm{Tx}$ and $\mathrm{Rx}$ antennas might also allow for the elimination of a T/R switch or duplex filter if enough isolation can be achieved between PA output and LNA input [5].

In order to quantify the filtering robustness of the antenna, we performed a system simulation using HP's Advanced Design System software to analyze system performance for both a conventional RF system architecture and a proposed RF system architecture based on the tunable SPA $\mathrm{Tx} / \mathrm{Rx}$ pair. The specific configuration we analyzed comprises 


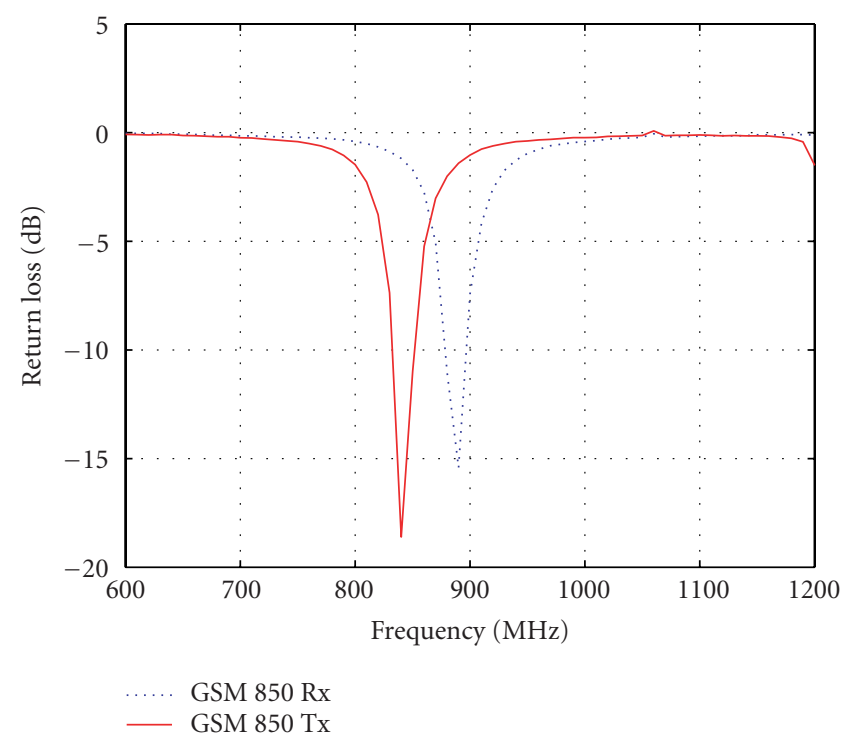

(a)

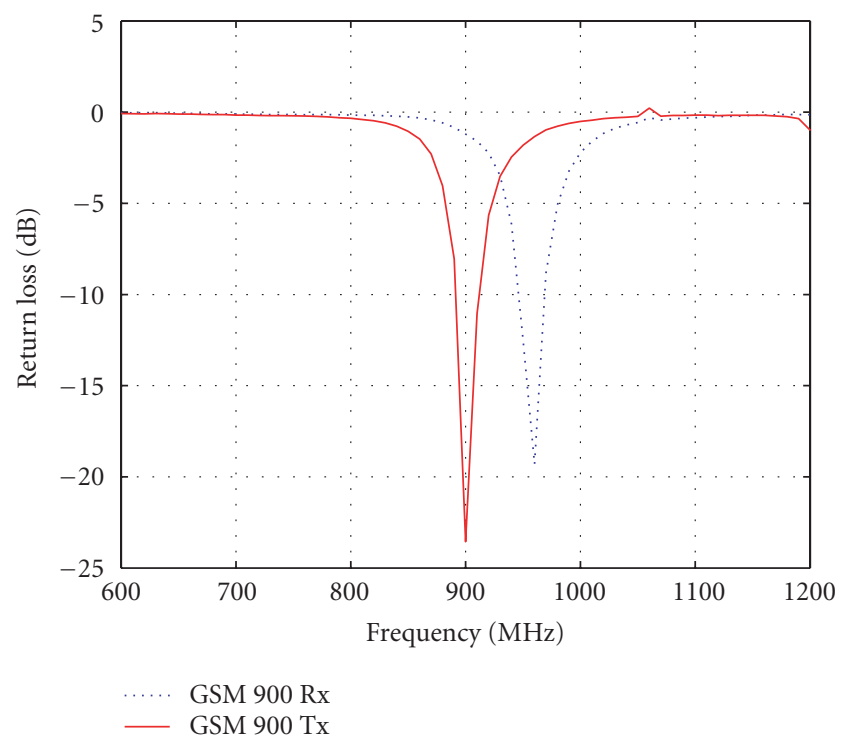

(b)

Figure 10: Measured return loss of tunable antenna pair tuned to (a) GSM 850 band and (b) GSM 900 band.

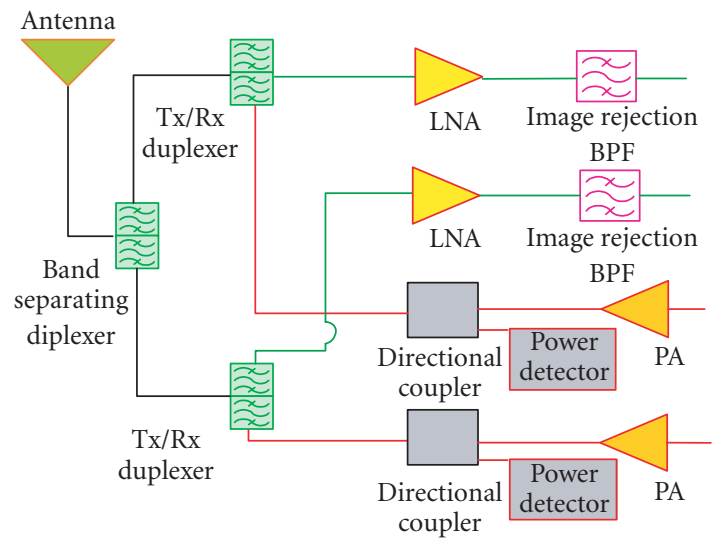

Figure 11: Conventional dual-band full-duplex RF front-end architecture.

a full duplex, dual-band (GSM 850 and PCS bands) superheterodyne receiver. We attempted to mimic the performance of off-the-shelf passive and active devices in our simulations. A block diagram of the conventional dual-band, full-duplex front end is shown in Figure 11. A block diagram of a proposed novel dual-band, full-duplex front end based on the reconfigurable antenna pair is shown in Figure 12.

The results of the simulations for the US cellular and PCS bands are summarized in Table 1. It should be noted that we obtained the antenna characteristics used in the simulation from a transmission line model [6]. If we had used measured data, the results would not have been as favorable for the image rejection. As can be seen, the architecture based on the reconfigurable antenna pair offers almost

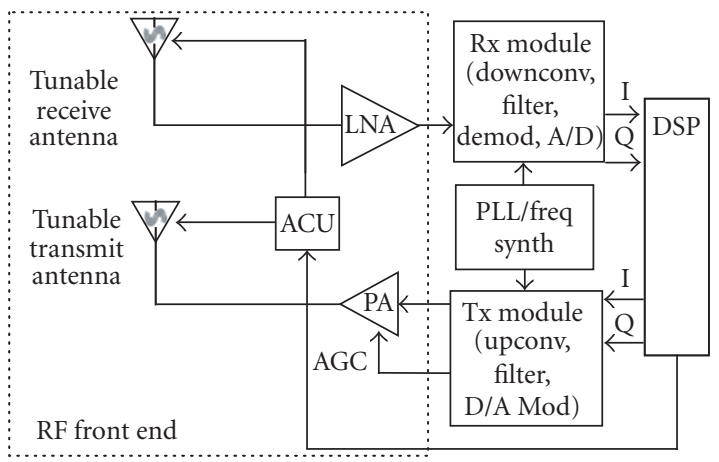

FIGURe 12: Proposed dual-band full-duplex RF front end implementing reconfigurable antenna pair.

the same image rejection as the conventional architecture while providing substantially better noise figure. Furthermore, the component count and cost of the SPA-pair architecture are substantial lower than that of the conventional architecture.

\section{FUTURE WORK}

The reconfigurable antennas that we have breadboarded to date all use PIN diode or FET switches. These switches are far from ideal and significantly reduce the radiation efficiency of the antennas. Furthermore, these devices also exhibit nonlinearities that contribute to harmonic and intermodulation distortions in both transmit and receive chains. However, RF MEMS switches appear poised to become 
TABLE 1: Comparison of simulated results of conventional and proposed RF front-end architectures.

\begin{tabular}{|c|c|c|c|c|}
\hline \multirow{2}{*}{$\begin{array}{l}\text { Architecture type } \\
\text { Band }\end{array}$} & \multicolumn{2}{|c|}{ Conventional architecture } & \multicolumn{2}{|c|}{ Proposed architecture } \\
\hline & GSM 850 & PCS & GSM 850 & PCS \\
\hline Desired signal at antenna & $-90 \mathrm{dBm}$ & $-90 \mathrm{dBm}$ & $-90 \mathrm{dBm}$ & $-90 \mathrm{dBm}$ \\
\hline Image (200 MHz offset) at ant. & $-60 \mathrm{dBm}$ & $-60 \mathrm{dBm}$ & $-60 \mathrm{dBm}$ & $-60 \mathrm{dBm}$ \\
\hline Desired signal at LNA out & $-82 \mathrm{dBm}$ & $-80 \mathrm{dBm}$ & $-77 \mathrm{dBm}$ & $-78 \mathrm{dBm}$ \\
\hline Undesired signal at LNA out & $-135 \mathrm{dBm}$ & $-120 \mathrm{dBm}$ & $-116 \mathrm{dBm}$ & $-110 \mathrm{dBm}$ \\
\hline Desired signal gain & $8 \mathrm{~dB}$ & $10 \mathrm{~dB}$ & $13 \mathrm{~dB}$ & $12 \mathrm{~dB}$ \\
\hline Undesired signal rejection & $75 \mathrm{~dB}$ & $60 \mathrm{~dB}$ & $56 \mathrm{~dB}$ & $50 \mathrm{~dB}$ \\
\hline System noise figure & $4.1 \mathrm{~dB}$ & $3.8 \mathrm{~dB}$ & $1.9 \mathrm{~dB}$ & $1.6 \mathrm{~dB}$ \\
\hline
\end{tabular}

viable alternatives in the near future to minimize these undesired effects. These switches are virtually ideal in terms of insertion loss and isolation, and their linearity is 40 to $50 \mathrm{~dB}$ better than GaAs devices [7, 8]. Currently, reliability and packaging issues concerning these devices are being resolved, and low-cost production techniques are being developed. Based on these developments, we expect to be able to evaluate the performance of reconfigurable antennas realized with RF MEMS switches in the near future. Implementing the reconfigurable antennas with MEMS technology will increase the performance and tuning range of an electronically small antenna. Hence, we expect that the two antenna pairs introduced in this paper can be replaced with a single antenna pair for all current wireless frequency bands including GSM, PCS, DCS, GPS, ISM, and so on.

\section{CONCLUSION}

A novel electronically tunable antenna pair and RF frontend architecture for current wireless communication bands (GSM 850/900, PCS, and DCS) were designed and demonstrated for SDR applications. Both high efficiency and small profile of the antenna were achieved by introducing a reconfigurable antenna pair that covers only the desired band instantaneously. Moreover, a narrowband filter-like response of the antenna pair drastically improves the SNR of RF front end and reduces the cost and size of current radio architectures by eliminating the need for a T/R switch or a duplex filter. Since the antenna pair is electronically reconfigured based on frequency information received from the DSPs, the proposed RF front-end architecture is a promising candidate for SDRs.

\section{ACKNOWLEDGMENTS}

The authors would like to thank Altera Corporation for donating the FPGA board to Arizona State University. The work described in this paper was supported by Connection One, an NSF-sponsored Industry University Cooperative Research Center.

\section{REFERENCES}

[1] P. Burns, Software Defined Radio for 3G, Artech House, Boston, Mass, USA, 2003.

[2] H. A. Wheeler, "Fundamental limitations of small antennas," Proc. IRE, vol. 35, pp. 1479-1488, December 1947.

[3] C. A. Balanis, Antenna Theory: Analysis and Design, John Wiley \& Sons, New York, NY, USA, 2nd edition, 1982.

[4] S. D. Rogers, J. T. Aberle, and D. T. Auckland, "Two-port model of an antenna for use in characterizing wireless communications systems obtained using efficiency measurements," IEEE Antennas Propagat. Mag., vol. 45, no. 3, pp. 115-118, 2003.

[5] D. T. Auckland, S. D. Rogers, and J. T. Aberle, "Reconfigurable antennas and RF front ends for portable wireless devices," in Proc. Software Defined Radio Technical Conference and Product Exposition (SDR '02), pp. 29-33, San Diego, Calif, USA, November 2002.

[6] R. Garg, P. Bhartia, I. Bahl, and A. Ittipiboon, Microstrip Antenna Design Handbook, Artech House, Boston, Mass, USA, 2003.

[7] E. R. Brown, "RF-MEMS switches for reconfigurable integrated circuits," IEEE Trans. Microwave Theory Tech., vol. 46, no. 11, pp. 1868-1880, 1998.

[8] G. M. Rebeiz, "RF MEMS for commercial and defense applications," presented at IEEE Phoenix Chapter of MTTS, November 2003.

Sung-Hoon Oh was born in Korea, in 1973. He received the B.S. degree in electrical engineering from the University of Tennessee, Knoxville, in 2000, and the M.S. degree in electrical engineering from the Ohio State University, Columbus, in 2002. From 2000 to 2002, he was a Graduate Research Associate at the ElectroScience Laboratory of the Ohio State University, where he was engaged in the development of highvoltage wideband transmission-line-transformer and pulse generator for plasma ramparts. He is currently pursuing the Ph.D. degree in electrical engineering from Arizona State University. From 2003 to the present, he has been with Connection One where he is a Graduate Research Assistant for Professor James T. Aberle. His main research interests include radio-frequency (RF) front-end designs for software-defined radios (SDRs) applications and reconfigurable antenna development for wireless communications. 
James T. Aberle received the B.S. and M.S. degrees in electrical engineering from Polytechnic Institute of New York (now Polytechnic University) in 1982 and 1985, respectively, and the Ph.D. degree in electrical engineering from the University of Massachusetts in 1989. He has been a faculty member at Arizona State University since 1989, where he is currently an Associate Professor of electrical engineering. His research interests include the design of radiofrequency systems for wireless applications as well as the modeling of complex electromagnetic phenomena. Dr. Aberle recently returned to ASU after a two-year leave of absence. During this leave Dr. Aberle worked for a startup company that provided technological solutions for the wireless market to increase functionality, improve performance, and decrease the cost of wireless equipment and systems.

Sreeraman Anantharaman received his BSEE from Arizona State University in December 2003. From March 2004 he has been employed at Rosum Corporation, Redwood City, Calif, as RF test and design validation Engineer. He is part of the testing and QA group at Rosum Corporation. His current focus is on development of RF and system level tests and test automation. While at Arizona State University, he was working with

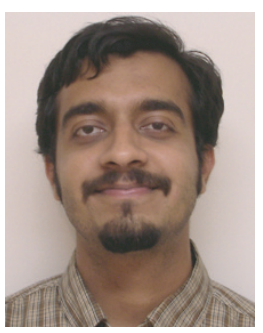
the Reconfigurable Antenna team in the Department of Electrical Engineering as part of the senior design project for the partial fulfillment of the BSEE degree. He is a member of IEEE.

Kentaro Arai was born in Japan in 1982. He received his B.S.E. in electrical engineering from Arizona State University in 2003. He is currently working for Hitachi-Omron Terminal Solutions which is located in Japan and dedicated to Automated Teller Machine Systems (4th largest ATM share company in the world) and other banking systems (including card reader, printing passbook, issuing card system), in Global Planning Unit. His main task is supporting and connecting foreign OEM and Japanese side. His desired career is working as a Leader of sales project team in the European and the U.S. markets.

Han Liang Chong was born in Penang, Malaysia, in 1981. He received his B.S.E. degree in electrical engineering from Arizona State University in 2003. Currently, he is pursuing a Master of Engineering degree in electrical engineering at Cornell University. His research interests include antennas, microwave devices, and radio frequency systems for wireless communications. He is currently working on Cornell's Ionospheric sCintillation Experiment CubeSat (ICE-CUBE) project.

Seng Chee Koay was born in Johore, Malaysia. He received his B.S.E degree (Cum Laude) in electrical engineering from Arizona State University in 2003. He is currently working as an Electrical Engineer at Chipset Engineering Group, Penang Design Center, Intel Corporation, Malaysia. His main tasks are developing scripts in Perl language, validation testing, and pico probing/electrical validation support for Intel chipsets. 